\title{
Finite Element Modeling of a Combustion Chamber with Premixed Laminar Flame using Lumped Parameter Analogy
}

\author{
Sadaf Shariati $^{1}$, Shahin Mohammadnejad ${ }^{1}$, Raimund Noske $^{2}$, Andreas Brockhinke ${ }^{2}$, Dirk Abel ${ }^{1}$ \\ ${ }^{1}$ Institute of Automatic Control, RWTH Aachen University \\ Steinbachstr. 54, 52074 Aachen, Germany \\ s.shariati@irt.rwth-aachen.de, shahin.mohammadnejad@studenti.polito.it \\ d.abel@irt.rwth-aachen.de \\ ${ }^{2}$ Faculty of Chemistry, Institute of Chemical Physics I, Bielefeld University, \\ 33501 Bielefeld, Germany \\ raimund.noske@uni-bielefeld.de, andreas.brockhinke@uni-bielefeld.de
}

\begin{abstract}
In this paper, the combustion chamber of a modern gas turbine with laminar premixed flame is modeled and its stability in different operating points is studied. The actuator in this set-up is a loudspeaker, which is modeled using AC/DC module in COMSOL Multiphysics, based on Lumped Parameter Analogy (LPA), and using Theile/Small parameters. The model is validated with the measurements in the cold flow. A so called $\sigma-\tau$ model is chosen as the flame model. Finally, the influence of different flame gains are studied on the thermo-acoustic stability of the system.
\end{abstract}

Keywords: Thermo-acoustic Instability, Finite Element Method, Stability analysis, Laminar Pre-mixed flame.

\section{Introduction}

Thermo-acoustic instabilities (TAI) are a common and yet crucial problems regarding modern gas turbines with the lean premixed flame, caused by an existing coupling between the unsteady heat release rate and the acoustic field inside the combustion chamber. Such combustion instabilities have adverse impacts on the system performance and its operating life [1-4]. The vibration generated by the TAI causes considerable fretting and wearing at the interfaces and fatigue cracking of combustor liner and consequently shorten the life of a combustor. Hence, modeling these instabilities, to be used in the prediction of their onset, have particular importance [6]. Large eddy simulation (LES) is a popular approach to model TAI. It is the mathematical model for turbulence flows used in computational fluid dynamics. This technique is among the most accurate approaches, because it models the flow field and the flame dynamic in the whole domain, but it requires enormous computational efforts [6]. Low-order network models are based on simplifying geometries by grouping components such as chambers, ducts and injectors and modeling each of them by a describing transfer matrix. These methods provide quick response. However, for complex geometries due to the lack of accuracy they are not applicable [7]. In order to eliminate some of the restrictions of the preceding methods, Finite Element Method (FEM) is employed. A useful feature of FEM is that it can be used in 3-D problems. Using FEM, the effects of the flame are incorporating into the acoustic wave equation as a monopole source of acoustic pressure perturbations through a so-called flame transfer function (FTF), which links the heat release fluctuations to the perturbations of the velocity at the burner inlet [8]. The resulting time dependent acoustic wave equation usually is converted to a complex eigenvalue problem in the frequency domain and is solved using FEM approach. Finally, the computed complex eigenvalues of the system are used for the stability analysis of the system in different operating points [10]. In the present work, the combustion chamber of a modern gas turbine is modeled using the commercial FEM software COMSOL Multiphysics. The system is identified experimentally and the stability of the system is analyzed in different operating points. The stability of the combustor is investigated by frequency response analysis and eigenvalues of the system.

\section{Description of the Experimental Set-Up}

The structure of the tube is depicted in Fig. 1, which is the presented in more details in [9]. The tube consists of the combustion chamber and the plenum, which are separated by a flame holder with the thickness of $35 \mathrm{~mm}$. The flame holder contains a slot with a width of $4 \mathrm{~mm}$ and the length of $60 \mathrm{~mm}$, through which the burning gas is blown into the combustion chamber. The pressure fluctuations in the plenum are recorded by two microphones, which are distributed axially in the plenum. The pressure fluctuation is recorded by the third microphone inside the combustion chamber. The internal medium of the system is actuated by a loudspeaker, which is mounted underneath the plenum.

\section{Governing equations}

As the flow velocity in the combustion chamber is lower than the speed of sound, it can be neglected. The inhomogeneous wave equation for the perturbations is then simplified by linearizing the Navier-Stokes equations as the following [2]:

$$
\begin{array}{cc}
T \partial^{2} p^{\prime} \\
c^{2} & \partial t^{2}
\end{array}-\nabla^{2} p^{\prime}=\begin{array}{cc}
\overline{\gamma-1} \partial q \\
c^{2} & \partial t
\end{array}
$$




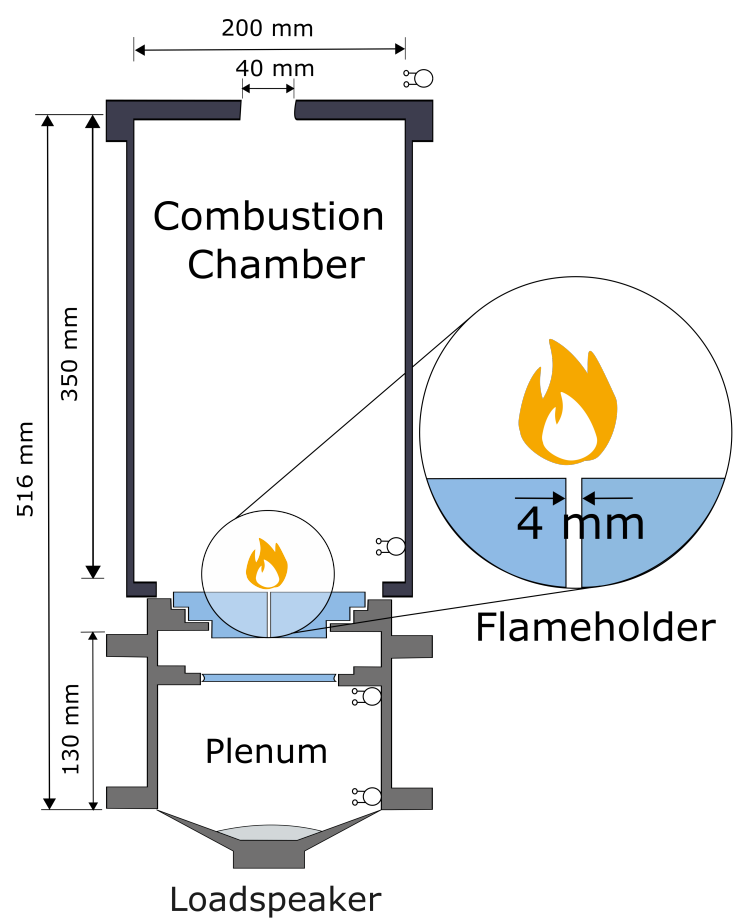

Fig. 1: Set-up of the combustion chamber.

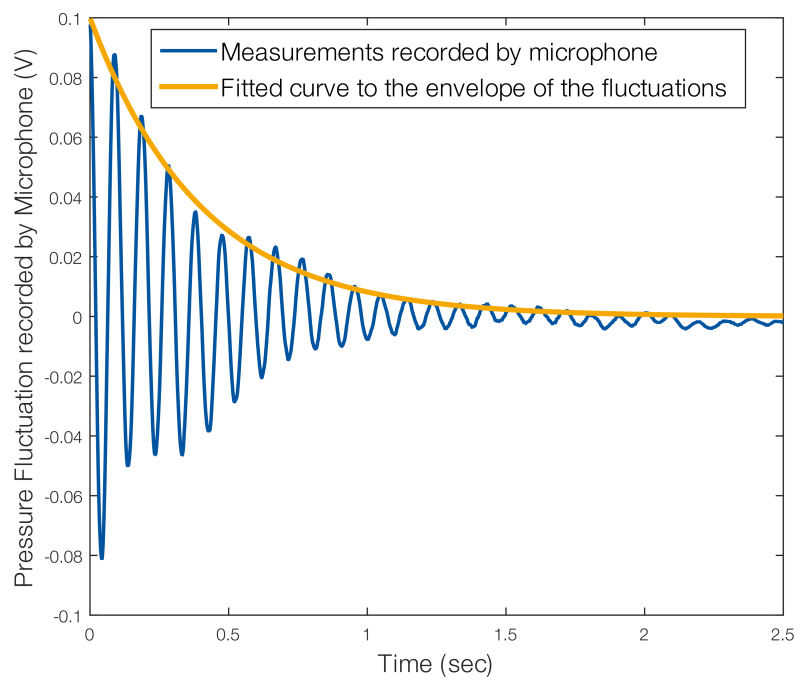

Fig. 2: The rate of decaying of the measured signal corresponds the damping coefficient of the set-up. Here the recorded pressure signal and its fitted envelope function with $\alpha=3 s^{-1}$ are depicted.

where $p^{\prime}, c$ and $\gamma$ are the pressure fluctuation, the speed of sound and the specific heats ratio, respectively. $q^{\prime}$ is the fluctuation of heat per unit volume. However, in this equation it is assumed that the medium is an ideal gas and the flow is inviscid. In [10], the viscous and thermal losses are modeled as the first derivative of $p^{\prime}$ multiplied by a damping factor $\alpha$ :

$$
\frac{1}{c^{2}} \frac{\partial^{2} p^{\prime}}{\partial t^{2}}+\frac{\alpha}{c^{2}} \frac{\partial p^{\prime}}{\partial t}-\nabla^{2} p^{\prime}=\frac{\gamma-1}{c^{2}} \frac{\partial q^{\prime}}{\partial t}
$$

in this work, the inhomogeneous Helmholtz equation, which is the final equation in FEM is considered [8]:

$$
\frac{\omega^{2}}{c^{2}} \hat{p}-\frac{i \omega \alpha}{c^{2}} \hat{p}+\nabla^{2} \hat{p}=-i \omega \frac{\gamma-1}{c^{2}} \hat{q}
$$

where:

$$
\begin{aligned}
p^{\prime} & =\hat{p}(\mathbf{x}) \exp (i \omega t) \\
q^{\prime} & =\hat{q}(\mathbf{x}) \exp (i \omega t)
\end{aligned}
$$

and $\omega$ is the complex eigenfrequency of the system and its real and imaginary parts correspond to the frequency and the growth rate of the oscillations, respectively. A negative imaginary part shows that the corresponding mode is unstable and the fluctuations grow with time. In the inhomogeneous Helmholtz equation, Eq. 3, $\alpha$ is the damping factor, obtained experimentally based on the Exponential Fit method explained in [11]. For the current system, the damping is found to be $\alpha=3 s^{-1}$ (Fig. 2).

\subsection{Flame model}

Experimental studies show that the density, the temperature and the pressure fluctuations in the premixed flames with low Mach number do not have such crucial roles in the heat fluctuation, as the velocity fluctuation does [12-14]. Hence, the flame transfer function (FTF) is simplified to the relationship between the relative heat release fluctuations and the relative velocity fluctuations in the upstream flame zone. However, choosing a suitable FTF for the flame is not straightforward. In the present study, according to the shape of the burner and the size of the flame, which is similar to Bunsen burners, a so called $\sigma-\tau$ model is preferred.

$$
F T F=\frac{\hat{q} \backslash \bar{q}}{\hat{u}_{i} \backslash \bar{u}_{i}}=e^{-\frac{1}{2} \omega^{2} \sigma^{2}} e^{-i \omega \tau}
$$

where $\bar{u}_{i}$ denotes the time average of the velocity upstream of the flame zone, $\tau=6 \mathrm{~ms}$ is the time delay between the injection and the ignition of the fuel, which has a Gaussian distribution in the time domain with a standard deviation $\sigma$ [15]. $\bar{q}$ indicates the time average of the heat release rate per unit area of the combustion chamber:

$$
\bar{q}=\rho_{i} \bar{u}_{i} c_{p}\left(T_{b}-T_{u}\right) \frac{A}{V}
$$


where $\rho_{i}$ is the gas density, $c_{p}$ is the specific heat at constant pressure, $T_{b}=2000 \mathrm{~K}$ is the temperature inside the combustion chamber, $T_{u}=288 \mathrm{~K}$ is the gas temperature inside the plenum, $A$ represents the cross-sectional area of the premixer and $V$ refers to the approximated volume of the flame zone. In Eq. 6, the expression $\exp \left(-\frac{1}{2} \omega^{2} \sigma^{2}\right)$ reflects the dependency of the transfer function gain on the frequency. Since flames are known to behave as a low-pass filter [3], the gain is supposed to fall in the higher frequencies. The cut-off frequency depends on the flame geometry, flow rate, equivalence ratio etc.

\section{Modeling the system in COMSOL Multiphysics}

To model the acoustic field inside the combustion chamber and mechanical and electrical characteristics of the loudspeaker, the Pressure Acoustic and AC/DC modules of COMSOL Multiphysics have been used, respectively. To model the loudspeaker, the so called Thiele/Small parameters are required, which are available by the manufacturer. The so called Lumped Parameter Analogy (LPA) approach is applied to model the loudspeaker in Comsol environment [16]. All the boundaries of the combustion chamber and the plenum are set to the sound hard boundaries, which means that the acoustic velocity is zero at these boundaries. The normal acceleration boundary is specified to the loudspeaker's membrane. The Perfectly Matched Layer (PML) boundary is defined at the exit of the combustion chamber and underneath of the system, where the loudspeaker is mounted. The PML is acting as an artificial boundary to limit the computational domain of the model [16].

\subsection{Modeling the loudspeaker}

The idea used in LPA, is based on the fact that the movement of the loudspeaker cone can be compared to the motion of a rigid piston in an infinite baffle [16]. Using the Electrical Circuit Interface within AC/DC module, the equivalent electrical and mechanical scheme of the loudspeaker shown in Fig. 3 and 4 can be modeled in COMSOL.
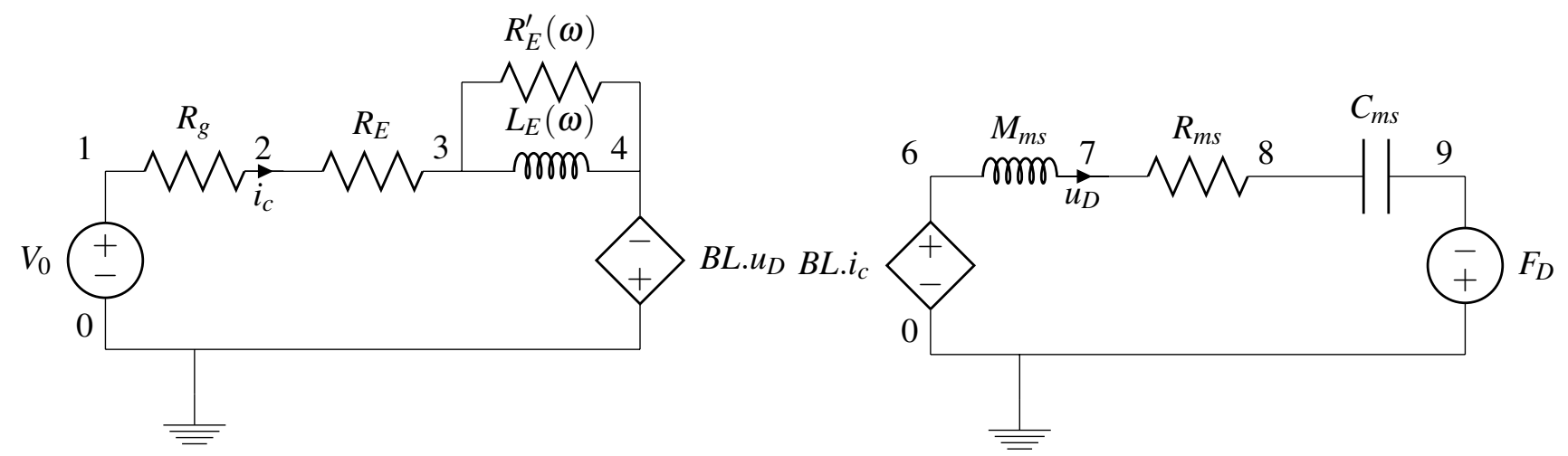

Fig. 3: Equivalent electrical circuit for the loudspeaker driver. Fig. 4: Equivalent mechanical circuit for the loudspeaker driver.

The parameters used in the equivalent circuits are listed in the table 1 . The voice coil inductance and the losses in it depend on the frequency and calculated by the following equations:

$$
\begin{aligned}
L_{E}(\omega) & =\left[\frac{L_{E}}{\sin \left(n_{c} \frac{\pi}{2}\right)}\right] \omega^{\left(n_{c}-1\right)} \\
R_{E}^{\prime}(\omega) & =\left[\frac{L_{E}}{\cos \left(n_{c} \frac{\pi}{2}\right)}\right] \omega^{n_{c}}
\end{aligned}
$$

The current controlled voltage source $B L \cdot u_{D}$ is the amount of the induced electromagnetic voltage, produced in the voice coil with length $L$, which moves with the velocity $u_{D}$ in the magnetic field $B$. As it is depicted in the circuits schemes, in the electrical circuit the current is donated by $i_{c}$ and in the mechanical circuit the velocity of the voice coil in the axial direction, $u_{D}$ considered as the current. $B L . i_{c}$ is the force acting on the loudspeaker cone and it is indeed the Lorentz force on a voice coil with the length of $L$ and the current $i_{c}$, subject to the magnetic flux $B$. At the end, $F_{D}$ expresses the pressure force acting on the loudspeaker cone and it is given by:

$$
F_{D}=\int\left(\Delta p n_{z}\right) d A
$$

In this expression, which couples the acoustic model to the lumped circuit model, $\Delta p$ is the pressure drop across the cone and $n_{z}$ is the axial component of the surface normal vector. Another argument used for the coupling between the acoustic module and AC/DC module, is the acceleration of the loudspeaker's membrane, computed as the following:

$$
\mathbf{a}=i \omega u_{D} \mathbf{e}_{z}
$$


Table 1: Description of the parameters and variables used in the speaker model.

\begin{tabular}{lll} 
Symbol & Description & Value \\
\hline$V_{0}$ & Voltage source & $0.05 \mathrm{~V}$ \\
$n_{c}$ & Voice coil loss factor & 0.2 \\
$R_{g}$ & Generator output resistance & $0 \Omega$ \\
$R_{E}$ & Voice coil DC resistance & $3.3 \Omega$ \\
$M_{m s}$ & Moving mass & $23 \mathrm{gr}$ \\
$R_{m s}$ & Suspension damping & $1.22 \mathrm{Ns} / \mathrm{m}$ \\
$C_{m s}$ & Suspension compliance & $4.98 \times 10^{-4} \mathrm{~m} / \mathrm{N}$ \\
$L_{E}(\omega)$ & Voice coil inductance & Variable \\
$R_{E}^{\prime}(\omega)$ & Losses in the voice coil & Variable \\
$B L . u_{D}$ & Current control voltage source & Variable \\
$B L . i_{c}$ & Lorentz force & Variable \\
$F_{D}$ & Voltage source & Variable
\end{tabular}

\subsection{Cold flow analysis}

To validate the model, the frequency response of the model without considering the unsteady heat release rate $(\hat{q}=0)$, known as Cold Flow Condition, is captured. Here, the system is excited harmonically by the loudspeaker in the range of $50 \mathrm{~Hz}$ to $550 \mathrm{~Hz}$. To identify the frequency response of the system using the experimental results, recorded by microphones, the correlation method is used, which is explained in details in $[9,17]$. The results are transferred to MATLAB using Comsol LiveLink for MATLAB, to plot frequency response of the system. In Fig. 5 the measured frequency responses of the system and the modeling results are compared. As it is depicted in Fig. 5, in the low frequency range, where the system is more prone to instability, the modeling and experimental results are matched.

The measured frequency modes inside the combustion chamber are in $90 \mathrm{~Hz}, 140 \mathrm{~Hz}, 300 \mathrm{~Hz}$ and $510 \mathrm{~Hz}$, corresponding to $565 \mathrm{rad} / \mathrm{sec}, 880 \mathrm{rad} / \mathrm{sec}, 1600 \mathrm{rad} / \mathrm{sec}$ and $3000 \mathrm{rad} / \mathrm{sec}$, respectively. The first significant resonance frequency inside the combustion chamber is not high enough to be considered as a longitudinal mode for the current geometry. On the other hand, as mentioned in [18], a large sudden area change in the connected tubes can serve as a Helmholtz resonator. To check this issue, the frequency of the possible Helmholtz mode is calculated as follows:

$$
f_{H}=\frac{c}{2 \pi} \sqrt{\frac{A_{3}}{A_{4} L_{3} L_{4, e f f}}}=90
$$

where $c$ is the sound speed, $A_{3}$ and $A_{4}$ are combustion chamber and exhaust port cross sectional area, respectively. $L_{3}$ is the length of the combustion chamber and $L_{4, \text { eff }}$ is the effective length of the exhaust port, computed as: $L_{4, e f f}=L_{4}+4 D / \pi, L_{4}$ and $D$ being the thickness and diameter of the exhaust port. Therefore, it can be concluded that combustion chamber in the present system acts as a Helmholtz resonator.

The dominant mode in the plenum, is roughly at $140 \mathrm{~Hz}$ (around $880 \mathrm{rad} / \mathrm{sec}$ ), which is the quarter wave of the combustion chamber:

$$
f_{1 / 4}=\frac{c}{4\left(L_{\text {total }}+L_{\text {correction }}\right)}=140
$$

where the $L_{\text {total }}$ is the total length of the tube, which must be corrected by a correction factor $L_{\text {correction }}$ [9].

\section{Stability analysis}

To determine the influence of the flame on the stability of the system, one possibility is to adjust the parameter $\sigma$ in the flame transfer function 6. In fact, modification of this parameter affects the gain of flame transfer function. Generally, increasing the standard deviation of the time delay reduces the impact of the flame response on the stability of the system, because it causes the enhancement of the low-pass character of the flame and consequently, leads to a larger stability margin. The variation of $\sigma$ is accomplished by defining a parametric sweep in the range of $1 \mathrm{~ms}$ to $8 \mathrm{~ms}$. It is observed that for $\sigma$ higher than $4 \mathrm{~ms}$, the system is always stable. Experiments show that the thermoacoustic instability in this set-up is due to the coupling between the flame and the acoustic mode at $90 \mathrm{~Hz}$ [17]. The frequency response of the system for two different values of $\sigma$ is shown in Fig. 6 . The positive phase shift for $\sigma=3 \mathrm{~ms}$ and negative phase shift for $\sigma=4 \mathrm{~ms}$ in the phase diagram at $90 \mathrm{~Hz}$, indicates that the corresponding mode is unstable in the first operating point and becomes stable in the second one.

\section{Stability analysis by studying the eigenfrequencies of the system}

In order to calculate eigenfrequencies of the system, the boundary conditions must be homogeneous. Therefore, the PML layers are removed from the both sides of the system, to study the eigenfrequency of the system. Moreover, in the position of the loudspeaker, the normal acceleration boundary condition should be substituted with the sound hard boundary, and at the exit of the combustion chamber, the sound soft boundary is introduced. $\sigma$ is swept by a parametric sweep function from $1 \mathrm{~ms}$ to $8 \mathrm{~ms}$ with the constant step size of $0.2 \mathrm{~ms}$. For each value of $\sigma$, the solver provides a new set of eigenvalues. The mode shapes of the system for the most predominant computed eigenfrequencies are shown in Fig. 8, 9 and 10. For the present system, in each set of eigenfrequencies, the second one is in the range of $90 \mathrm{~Hz}$. It is observed that the imaginary part of the eigenfrequency at $90 \mathrm{~Hz}$ has the most dependency to the magnitude of $\sigma$. The 

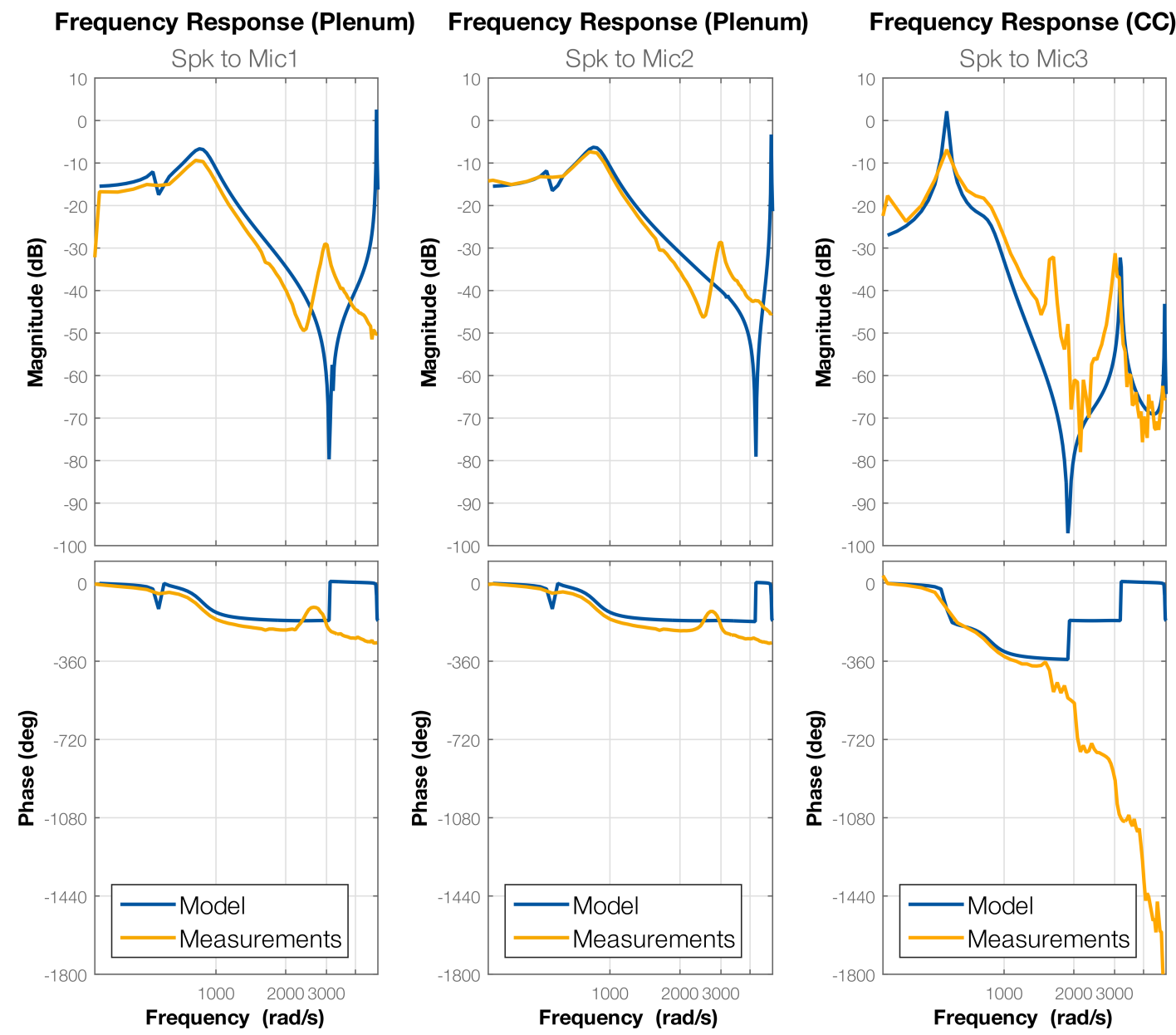

Fig. 5: Frequency response of the system in cold flow condition.

variation of the real and the imaginary part of the eigenfrequency at $90 \mathrm{~Hz}$ for some selective values of $\sigma$ is depicted in table 6 . In Fig. 7, it is shown that for $\sigma>4.6 \mathrm{~ms}$, the growth rate becomes negative, therefore, system is completely stable.

\begin{tabular}{ccc}
\hline \multicolumn{3}{c}{ Frequency } \\
\hline$\sigma$ & Frequency of oscillations Hz & Growth rate $-\operatorname{Im}(\omega)[1 / \mathrm{s}]$ \\
\hline 1 & 82.44 & 21 \\
2 & 85 & 14.5 \\
3 & 85.8 & 8.4 \\
4 & 88.3 & 1.3 \\
5 & 91 & -0.08 \\
6 & 91.5 & -1 \\
\hline
\end{tabular}



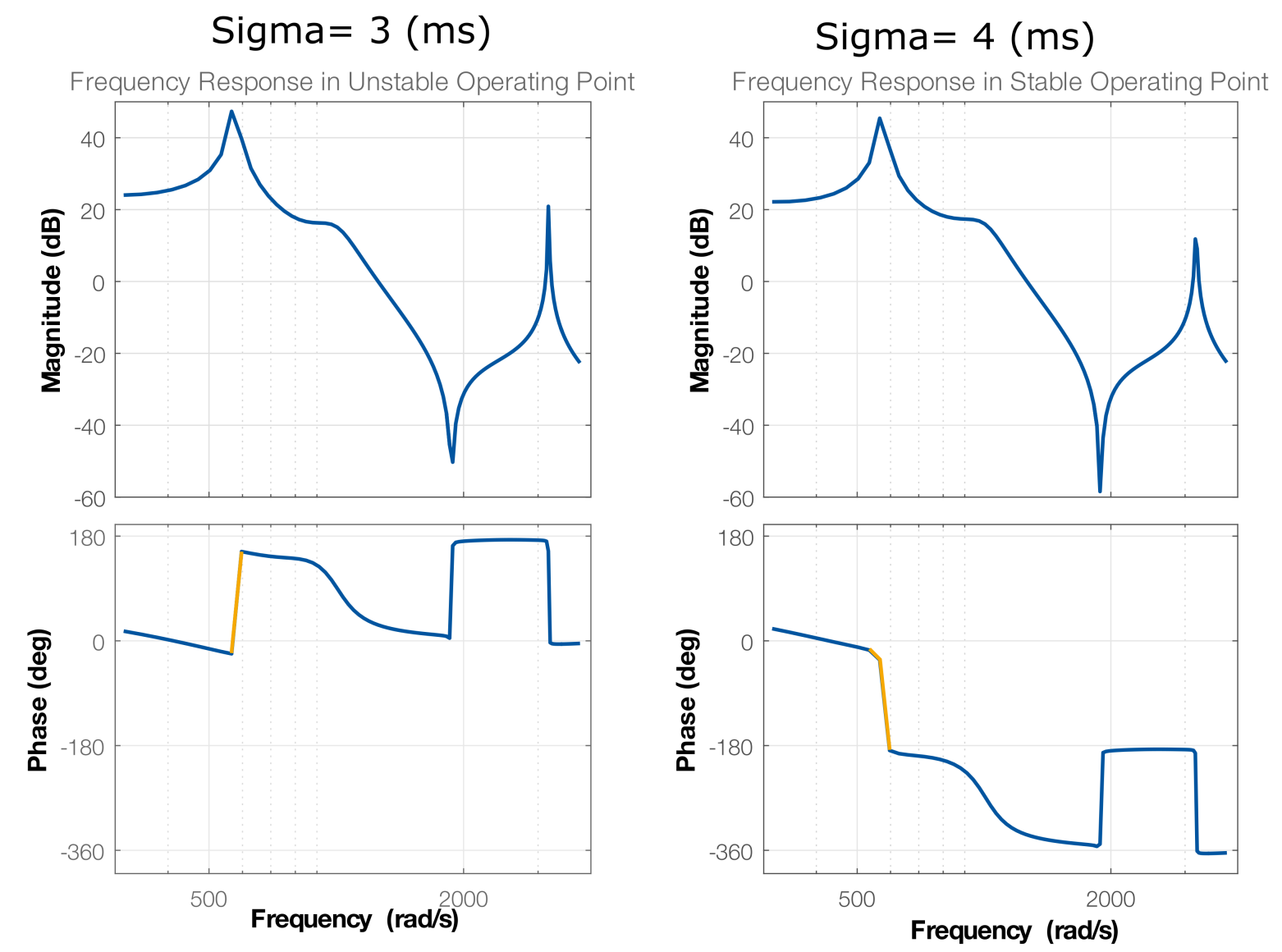

Fig. 6: Frequency Response of the system in the stable and unstable operating points.

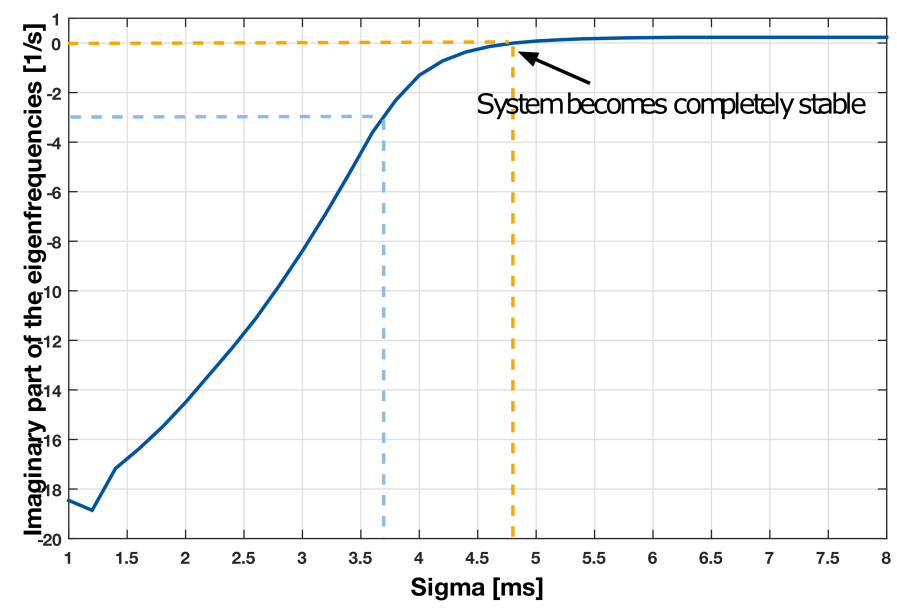

Fig. 7: Variation of the imaginary part of the eigenvalue at $90 \mathrm{~Hz}$. 


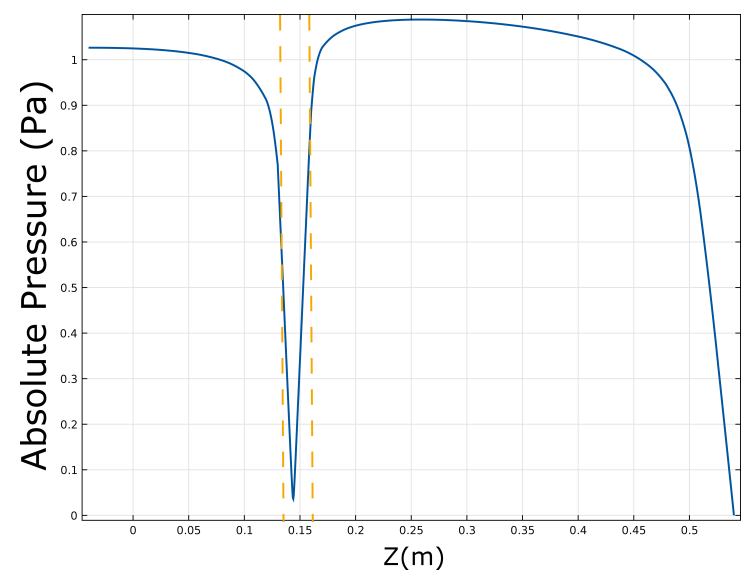

Fig. 8: Mode shape at $90 \mathrm{~Hz}$.

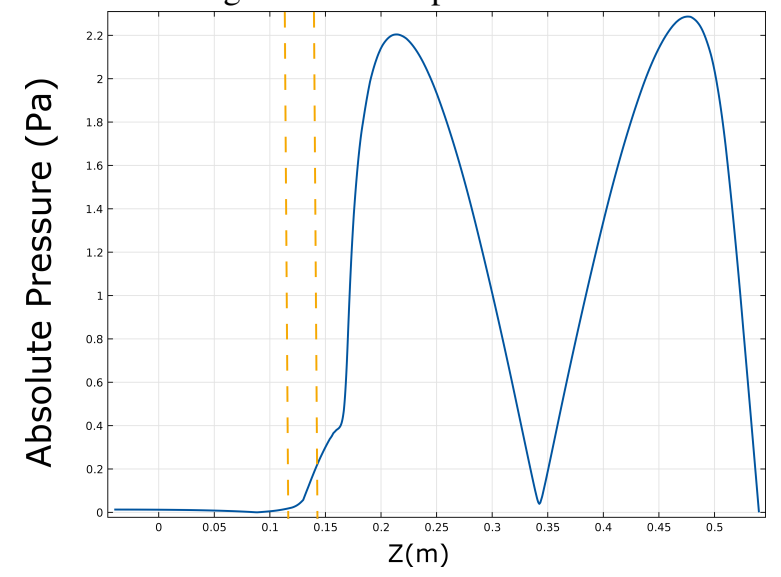

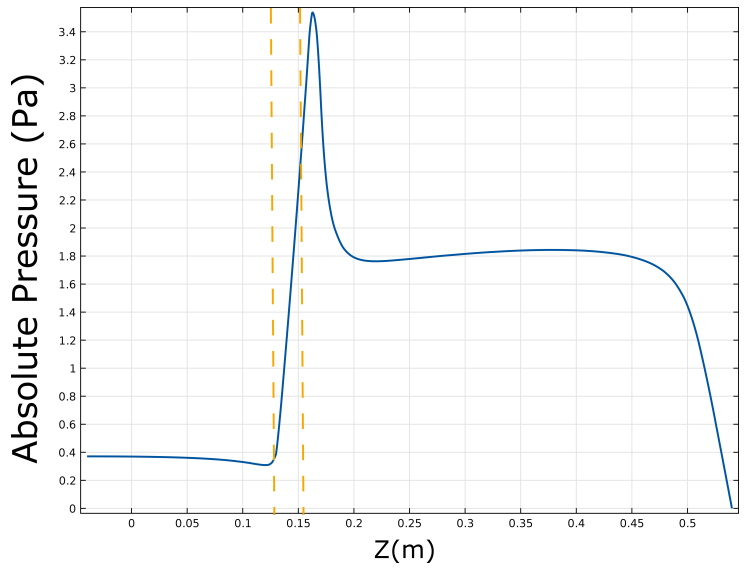

Fig. 9: Mode shape at $250 \mathrm{~Hz}$.

Fig. 10: Mode shape at $500 \mathrm{~Hz}$.

\section{Conclusion}

A finite element method has been applied to model the thermoacoustic instability in a combustion chamber, which a loudspeaker has been mounted underneath of it. To validate the model, the resulting frequency response is compered to the experiments in the cold flow condition, which fits the measurements in the low frequencies and shows more mismatch in the higher frequency range. Considering the characteristics of the flame in the system, a $\sigma-\tau$ FTF has been used to investigate the influence of the flame on the stability of the system. Employing two different approaches for the stability analysis showed that, the stability of the system around Helmholtz resonance frequency is sensible to the magnitude of the gain of FTF. The system moved from instability to stability, when $\sigma$ changed from $3 \mathrm{~ms}$ to $4 \mathrm{~ms}$.

\section{References}

[1] D. U. Campos-Delgado, B. H. Schuermans, K. Zhou, C. O. Paschereit, E. A. Gallestey, and A. Poncet, "Thermoacoustic instabilities: modeling and control," Control Systems Technology, IEEE Transactions on, vol. 11, no. 4, pp. 429-447, 2003.

[2] A. P. Dowling, "Nonlinear self-excited oscillations of a ducted flame," Journal of Fluid Mechanics, vol. 346, pp. 271-290, 1997.

[3] A. P. Dowling and S. R. Stow, "Acoustic Analysis of Gas Turbine Combustors," Journal of Propultion And Power, vol. 19, no. 5, pp. 751-764, 2003.

[4] T. C. Lieuwen, "Investigation of combustion instability mechanisms in premixed gas turbines," Ph.D. thesis, Georgia Institute of Technology, 1999.

[5] S. M. Camporeale, B. Fortunato, and G. Campa, "A Finite Element Method for Three-Dimensional Analysis of Thermo-acoustic Combustion Instability," Journal of Engineering for Gas Turbines and Power, 2011.

[6] G. Campa, "Prediction of the Thermoacoustic Combustion Instability in Gas Turbines," 2012.

[7] S. Evesque and W. Polifke, "Low-order acoustic modelling for annular combustors: Validation and inclusion of modal coupling," in Proceedings of ASME Turbo Expo 2002: Power for Land, Sea, and Air, 2002, pp. 321-331.

[8] G. A. Mensah and J. P. Moeck, "EFFICIENT COMPUTATION OF THERMOACOUSTIC MODES IN ANNULAR COMBUSTION CHAMBERS BASED ON BLOCH-WAVE THEORY," in Proceedings of ASME Turbo Expo 2015: Turbine Technical Conference and Exposition, 2015. 
[9] S. Shariati, A. A. da Franca, B. Oezer, R. Noske, D. Abel, and A. Brockhinke, "Modeling and model predictive control of combustion instabilities in a multi-section combustion chamber using two-port elements," in Proceedings of Control Applications (CCA), 2014 IEEE Conference on, 2014, pp. 2108-2113.

[10] D. Laera, S. M. Camporeale, and G. Campa, "Application of a Weakly Non-Linear Analysis to the Analysis of Thermoacoustic Combustion Instabilities," in Proceeding of the 2014 COMSOL Conference in Cambridge, 2014.

[11] M. Wagner, C. Jörg, and T. Sattelmayer, "Comparison of the accuracy of time-domain measurement methods for combustor damping," in Proceeding of the ASME Turbo Expo 2013: Turbine Technical Conference and Exposition, 2013, pp. V01AT04A064V01AT04A064.

[12] P. R. Alemela, D. Fanaca, F. Ettner, C. Hirsch, T. Sattelmayer, and B. Schuermans, "Flame transfer matrices of a premixed flame and a global check with modelling and experiments," in Proceeding of the ASME Turbo Expo 2008: Power for Land, Sea, and Air, 2008, pp. 11-19.

[13] P. A. Hield, M. J. Brear, and S. H. Jin, "Thermoacoustic limit cycles in a premixed laboratory combustor with open and choked exits," Combustion and Flame, vol. 156, no. 9, pp. 1683-1697, 2009.

[14] T. Lieuwen, "Modeling premixed combustion-acoustic wave interactions: A review," Journal of Propulsion and Power, vol. 19, no. 5, pp. 765-781, 2003.

[15] B. Schuermans, V. Bellucci, F. Guethe, F. Meili, P. Flohr, and C. O. Paschereit, "A detailed analysis of thermoacoustic interaction mechanisms in a turbulent premixed flame," in Proceeding of the ASME Turbo Expo 2004: Power for Land, Sea, and Air, 2004, pp. 539-551.

[16] COMSOL Multiphysics, "COMSOL multiphysics user guide (Version 4.3 a)," COMSOL, AB, 2012.

[17] S. Shariati, A. Adamski, R. Noske, A. Brockhinke, and D. Abel, "Modelling a Combustion Chamber acting as a Helmholtz Resonator using the Two-Microphones-Method and Design of a LQR," in Proceeding of the Control Applications (CCA), 2015 IEEE Conference on, 2015.

[18] T. Poinsot and D. Veynante, Theoretical and Numerical Combustion. R.T. Edwards Inc., 2005. 